\title{
Adherencias abdominales postoperatorias: patogénesis y técnicas actuales de prevención
}

\author{
Postoperative abdominal adhesions: pathogenesis and current preventive techniques \\ Gilberto Guzmán-Valdivia-Gómez ${ }^{1 *}$, Eduardo Tena-Betancourt² y Patricio Martínez de Alva-Coria ${ }^{1}$ \\ ${ }^{1}$ Unidad de investigación; 'Bioterio y Cirugía Experimental. Facultad Mexicana de Medicina, Universidad La Salle, Ciudad de México, México
}

\section{Resumen}

Las adherencias abdominales postoperatorias (AAP) ocurren en más del 90\% de los pacientes sometidos a cirugía de abdomen; pueden causar dolor abdominal crónico, infertilidad femenina y obstrucción intestinal recurrente, que requieren intervenciones quirúrgicas que son causa de morbilidad y mortalidad, así como altos costos. La formación de AAP se debe a un desequilibrio entre la fibrinogénesis y la fibrinólisis a favor de la primera, asociada con hipoxia tisular secundaria a la agresión del peritoneo, también debido a la propia respuesta inflamatoria y al aumento en la población de fibroblastos de adherencia que inhiben la degradación de la matriz extracelular y facilitan el colágeno maduro y el tejido conectivo de sostén. La prevención de las AAP disminuye las complicaciones secundarias, así como la necesidad de hospitalizaciones y cirugías, y en consecuencia se conseguirá una contención de costos. Conocer el proceso fisiopatológico de las AAP permite establecer estrategias de investigación para poder intervenir en su formación y prevenirlas en los diferentes pasos del proceso.

PALABRAS CLAVE: Adherencias de tejido. Cavidad abdominal. Cirugía. Peritoneo. Reintervenciones.

\begin{abstract}
Post-operative abdominal adhesions (PAA) are a condition that occurs in more than $90 \%$ of patients undergoing abdomen surgery; they can cause chronic abdominal pain, female infertility and repeated bowel obstruction, requiring repetitive surgical interventions causing morbidity and mortality, as well as high costs. The formation of the PAA is due to an imbalance between the fibrinogenesis and fibrinolysis in favor of the first, associated with tissue hypoxia secondary to aggression of the peritoneum, also due to the own inflammatory response and the increase in the population of adhesion fibroblasts which inhibit the degradation of the extracellular matrix and facilitate mature collagen and supporting connective tissue. The prevention of PAA will decrease secondary complications, as well as hospitalizations, surgeries and consequently, cost containment. The PAA pathophysiologic process allows establishing research strategies in order to prevent them.
\end{abstract}

KEY WORDS: Tissue adhesions. Abdominal cavity. Surgery. Peritoneum. Reoperation.

\author{
Correspondencia: \\ *Gilberto G. Valdivia-Gómez \\ Fuentes, 17 \\ Del. Tlalpan \\ C.P. 14000 , Ciudad de México, México \\ E-mail: gilberto.guzmanvaldivia@ULSA.mx
}

Fecha de recepción: 06-06-2018

Fecha de aceptación: 20-09-2018

DOI: $10.24875 / C I R U .18000511$
Cir Cir. 2019;87:698-703 www.cirugiaycirujanos.com 


\section{Introducción}

Las adherencias abdominales son puentes vascularizados e inervados de tejido conectivo formados al azar entre las asas intestinales, el peritoneo y la pared abdominal'. Son una reacción fisiológica a la agresión de los tejidos que puede llevar a reintervenciones quirúrgicas, dolor abdominal crónico, obstrucción intestinal e infertilidad en las mujeres ${ }^{2-4}$. También se ha descrito que hasta el $93 \%$ de los pacientes que han tenido una o más cirugías desarrollan adherencias abdominales postoperatorias $(\mathrm{AAP})^{5}$, mientras que hasta el $15-20 \%$ de la infertilidad femenina se produce como resultado de estas ${ }^{4}$. La importancia de entender la patogénesis de las AAP es encontrar posibles métodos de prevención de su formación, al intervenir en los diferentes momentos durante la formación de adherencias, así como reconocer las proteínas que serán útiles como marcadores en el seguimiento de la formación de adherencias en estudios experimentales y en diversos modelos.

Las AAP son el resultado de una lesión del peritoneo visceral o parietal, que está formado por células mesoteliales. La lesión inicial puede producirse por su corte o incisión, electrocoagulación, contacto con suturas y cuerpos extraños, ante los que reacciona formando abundante tejido cicatricial aberrante ${ }^{6,7}$. Después de la lesión de las células mesoteliales se produce una liberación de sustancias vasoactivas y cininas (citocinas), producidas principalmente por los mastocitos que actúan en el sitio de la lesión, lo que lleva a vasodilatación y aumento de la permeabilidad vascular, formando así un exudado rico en fibrina ${ }^{6,8-10}$. Durante la reparación normal del peritoneo, la reacción morfológica inicial de la serosa se produce dentro de las primeras 12 horas, en las que aparece una densa capa de fibrina infiltrada con leucocitos polimorfonucleares; entre las 24 y las 36 horas de iniciado el proceso aparecen macrófagos sobre la capa de fibrina. En este momento pueden observarse células mesoteliales en la profundidad de este exudado que durante el proceso irán ascendiendo. Por el día 5, cuando el proceso de reparación se ha completado parcialmente, ya existe una capa simple de células mesoteliales. En el día 8, estas células mesoteliales se encuentran firmemente unidas a la membrana basal del segmento lesionado ${ }^{7,8,10}$. Estos serían los simples cambios morfológicos del peritoneo dañado que vienen dados por la activación o la inhibición de proteínas complejas de reparación.
La fibrina presente en este exudado inicial de reparación es el resultado final del proceso de la cascada de la coagulación, y al mismo tiempo hay una proliferación concomitante de fibroblastos encargados de la formación de una matriz extracelular y colágeno que favorecen la formación de tejido cicatricial, que es el primer esbozo de las adherencias. Posteriormente se produce un aumento de la expresión del factor de crecimiento vascular endotelial, que lleva a la proliferación de células endoteliales y a la formación tardía de las estructuras vasculares de las adherencias ${ }^{8,10}$. La matriz extracelular es el precursor de la reparación del tejido conectivo y está compuesta principalmente por fibroblastos fusiformes que sintetizan colágeno maduro, fibronectina y fibrina (esta última proteína procede de la activación de la cascada de la coagulación), que se comportan como tejidos de apoyo. La activación de los fibroblastos para sintetizar colágeno y fibronectina, así como su migración a la matriz extracelular, vienen dadas por factores de crecimiento sintetizados por las plaquetas, los polimorfonucleares, las células endoteliales dañadas y los macrófagos (Tabla 1).

Se ha demostrado ${ }^{4}$ que los fibroblastos que originan adherencias tienen un fenotipo diferente del de los fibroblastos comunes encontrados en el tejido peritoneal; tal transformación de fenotipo se ha relacionado con hipoxia tisular. Estos fibroblastos de adherencia tienen un aumento en la expresión de mRNA de colágeno tipo I, fibronectina, metaloproteinasa-1, inhibidores de tejido de metaloproteinasas, factor de crecimiento tisular beta 1, ciclooxigenasa 2 (COX-2) e interleucina 10; estas proteínas están en favor de la formación de adherencias al actuar en distintos momentos (Fig. 1).

La COX-2 es una enzima que regula la angiogénesis en el proceso inflamatorio de la formación de AAP. En presencia de hipoxia tisular o de fibroblastos de adherencia, aumenta la expresión de COX-28. Durante la formación de AAP densas, el sistema fibrinolítico desempeña un papel fundamental para degradar la fibrina formada en el sitio de la lesión por convertir el plasminógeno en plasmina, que es en última instancia la encargada de degradar la fibrina. La conversión de plasminógeno en plasmina se determina por el activador tisular del plasminógeno (tPA) y el activador de plasminógeno tipo urocinasa (UPA) $)^{1,6,8-11}$; ambos se expresan por el complejo de las células endoteliales y mesoteliales, por macrófagos y por fibroblastos.

Se ha observado que, en la cavidad peritoneal, el tPA es el causante de hasta el $95 \%$ de la acción activadora 
Cirugía y Cirujanos. 2019;87

Tabla 1. Factores de crecimiento relacionados con la reparación del peritoneo

\begin{tabular}{|c|c|c|}
\hline Factor & Origen & Acción \\
\hline Factor de crecimiento epidérmico (EGF) & $\begin{array}{l}\text { Macrófagos, células epiteliales y } \\
\text { plaquetas }\end{array}$ & $\begin{array}{l}\text { Estimula la producción de epitelio y activa células } \\
\text { inflamatorias y fibroblastos }\end{array}$ \\
\hline Factor de crecimiento transformante alfa (TGF- $\alpha$ ) & $\begin{array}{l}\text { Macrófagos, neutrófilos y } \\
\text { plaquetas }\end{array}$ & $\begin{array}{l}\text { Activa fibroblastos y promueve angiogénesis } \\
\text { (proliferación, síntesis de colágena y colagenasa) }\end{array}$ \\
\hline Factor de crecimiento fibroblástico (FGF) & Macrófagos y células epiteliales & Migración fibroblástica (quimiotaxis) \\
\hline Factor de crecimiento transformante beta (TGF- $\beta$ ) & Macrófagos y plaquetas & $\begin{array}{l}\text { Estimula angiogénesis y migración de neutrófilos, } \\
\text { macrófagos y fibroblastos a la matriz extracelular }\end{array}$ \\
\hline $\begin{array}{l}\text { Factor de crecimiento derivado de las } \\
\text { plaquetas (PDGF) }\end{array}$ & $\begin{array}{l}\text { Plaquetas, macrófagos, } \\
\text { fibroblastos y células endoteliales }\end{array}$ & $\begin{array}{l}\text { Estimula fibroblastos, proliferación y quimiotaxis a la } \\
\text { matriz extracelular }\end{array}$ \\
\hline Sustancia P de taquicinina (SP) & $\begin{array}{l}\text { Macrófagos, plaquetas y } \\
\text { neutrófilos }\end{array}$ & $\begin{array}{l}\text { Vasodilatación, quimiotaxis, activación de fibroblastos } \\
\text { y producción de interleucinas proinflamatorias }\end{array}$ \\
\hline
\end{tabular}

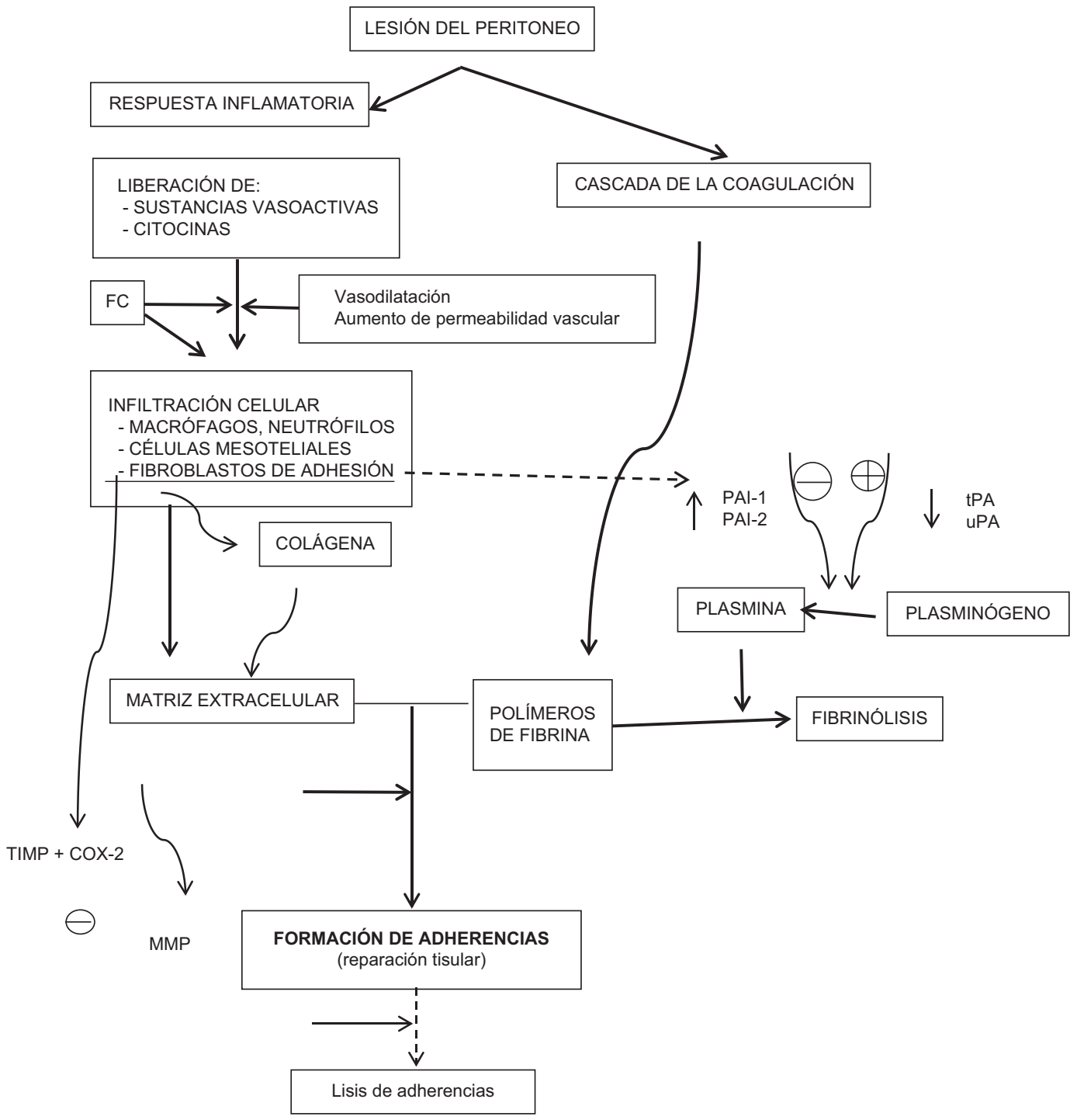

Figura 1. Fisiopatología de la formación de adherencias abdominales. La formación de adherencias abdominales postoperatorias se inicia con la lesión del peritoneo y la respuesta inflamatoria asociada a la activación de la cascada de la coagulación, que favorece la formación excesiva de depósitos de fibrina durante el proceso de reparación, bajo una fibrinólisis inefectiva.

FC: factor de crecimiento; MMP: metaloproteinasas (colagenasas); PAl: inhibidor del activador del plasminógeno; TIMP: inhibidor tisular de metaloproteinasas; $t P A$ : activador tisular del plasminógeno; uPA: activador del plasminógeno tipo urocinasa. 
$y$, en consecuencia, de la degradación de la fibrina ${ }^{1,6}$, mientras que el uPA desempeña un papel más importante durante la remodelación tisular, porque actúa degradando los componentes de la matriz extracelular. Además, como proceso equilibrante, la activación del plasminógeno es inhibida por el factor inhibidor del activador del plasminógeno (PAl) tipo 1 y tipo 2, aunque la glucoproteína de PAl-1 tiene una mayor actividad inhibitoria. Ambos activadores del plasminógeno, PAI-1 y PAI-2, son producidos por las células endoteliales y mesoteliales, los fibroblastos, los monocitos y los macrófagos presentes. El PAl-1 se considera un factor importante en la fisiopatología de las AAP, y se encuentra en altas concentraciones en los pacientes con adherencias peritoneales'.

Por otra parte, en los fibroblastos comunes, la relación tPA/PAl-1 es un 80\% mayor que en los fibroblastos de adherencia, lo que favorece la fibrinólisis. Durante un evento hipóxico, la relación tPA/PAI-1 disminuye más del $90 \%$ en los fibroblastos comunes, y se observa una disminución aún mayor en los fibroblastos de adherencia; la disminución en esta relación trae como consecuencia una menor acción fibrinolítica y una mayor formación de AAP.

Teniendo en cuenta la complejidad de los mecanismos que desencadenan la formación de AAP, su génesis puede resumirse como el resultado final de un desequilibrio entre el proceso de fibrogénesis y de fibrinólisis en favor de la primera, junto con hipoxia tisular secundaria al daño mesotelial, mayor respuesta inflamatoria del tejido lesionado y un aumento de la población de fibroblastos de adherencia y de la inhibición de la degradación de la matriz extracelular.

El objetivo del presente artículo es revisar los eventos fisiológicos que intervienen en la formación de AAP y definir los sitios de oportunidad de intervención para prevenir su formación. En este fenómeno de formación de AAP, los estudios experimentales deben centrarse en las diferentes etapas de la cascada de la coagulación para intentar reducir la formación de fibrina y su organización, así como en la maduración del colágeno y su degradación, para poder influir en los pasos involucrados en el proceso de la inflamación: vasodilatación y aumento de la permeabilidad vascular, disminución en la concentración de fibrina y sus precursores a través de la inhibición de la COX-2 y fomentando la acción de los activadores del plasminógeno, y limitación de la función de los factores inhibidores de la activación del plasminógeno.

\section{Prevención}

Se han diseñado numerosas estrategias en la búsqueda de métodos dirigidos a disminuir la formación de AAP, basadas en datos específicos relacionados con el origen fisiopatológico de estas. A continuación se comentan varios enfoques ${ }^{3,12}$.

\section{La técnica quirúrgica}

El manejo atraumático de los tejidos durante la cirugía trata de disminuir el daño al peritoneo parietal y visceral (mesotelio), y de limitar la hipoxia local ${ }^{2}$. Hay dos principios básicos dirigidos a prevenir la formación de AAP: evitar las incisiones en los tejidos muy vascularizados (p. ej., el músculo) y minimizar el trauma quirúrgico, incluyendo el abuso de la electrocoagulación ${ }^{13}$.

Los especialistas en microcirugía han aceptado que el manejo de los tejidos blandos con guantes es menos traumático que el contacto con instrumentos quirúrgicos ${ }^{2}$. En modelos animales se ha comprobado que el tiempo quirúrgico y el sangrado perioperatorio aumentan la incidencia de AAP al haber mayor daño tisular inducido ${ }^{14}$. La mayoría de los estudios coinciden en que la laparoscopia reduce de manera importante la formación de AAP en comparación con la laparotomía abierta ${ }^{3,15-17}$.

\section{Profilaxis farmacológica}

Los antinflamatorios no esteroideos inhiben la COX1 y la COX-2, y con ello impiden la síntesis de prostaglandinas y de tromboxanos ${ }^{2}$. Los estudios en animales han reportado que el uso de estos medicamentos disminuye la formación de AAP. En las ratas, por ejemplo, un inhibidor selectivo de la COX-2 vertido sobre el peritoneo reduce la formación de adherencias peritoneales ${ }^{18-20}$.

Se han comparado los inhibidores selectivos de la COX-2 (nimesulida, celecoxib y parecoxib) ${ }^{21-23}$, tanto en aplicación intraperitoneal como intramuscular, y se ha observado una reducción en la formación de AAP, sin diferencias significativas relacionadas con la vía de aplicación; sin embargo, no hay evidencia significativa para recomendar su uso en seres humanos y hacen falta estudios prospectivos al respecto ${ }^{13,21}$.

La heparina es un ácido aniónico de glucosaminoglicano que se une a la antitrombina III (AT-III) para formar el complejo heparina-AT-III e inhibir así la activación de los factores de la coagulación IX, X, XI y 
XII, previniendo la formación de fibrina. Un estudio en ratas de laboratorio demostró que la administración de heparina combinada con carboximetilcelulosa, un agente que actúa como barrera, en diferentes dosis $(62,5,125$ y $250 \mathrm{UI})$, resultó eficaz y segura, y se obtuvieron los mejores resultados cuando se aplicó la mayor dosis de heparina ${ }^{1,24-26}$. Sin embargo, la dosis óptima necesaria para limitar la formación de adherencias todavía no ha sido completamente definida, por lo que es necesario identificar la dosis efectiva para la prevención de la formación de adherencias, pero sin aumentar el riesgo de sangrado.

\section{Otras terapias}

Como terapia alternativa se ha propuesto el uso de barreras mecánicas que eviten el contacto entre las superficies de células mesoteliales y así impedir la activación de los procesos inflamatorios y de la cascada de la coagulación'. Se ha propuesto que las barreras mecánicas reúnan las siguientes condiciones: no crear reacción de rechazo, ser bioabsorbibles y ser fáciles de manipular ${ }^{9}$. Existen barreras mecánicas de consistencia líquida, entre ellas el dextrano 70 , una solución hipertónica que se cree que puede actuar en el recubrimiento de superficies denudadas y alterar la estructura de la fibrina, haciéndola más susceptible a la lisis ${ }^{27}$. Sin embargo, su uso ha sido abandonado porque no demuestra eficacia clínica suficiente $^{1,12}$. Entre las barreras mecánicas sólidas, las más representativas son el glicol de polietileno (SparyGel $\left.{ }^{\circledR}\right)$, la hoja de celulosa oxidada regenerada (Interceed $^{\circledR}$ ) y la carboximetilcelulosa junto con hialuronato de sodio (Seprafilm ${ }^{\circledR}$ ); las dos últimas están aprobadas por la Food and Drug Administration de los EE.UU. Estas barreras se han empleado principalmente en cirugía ginecológica y pélvica, y aunque han demostrado cierta eficacia para disminuir la gravedad o la extensión de las AAP entre las asas del intestino y la pared abdominal, no han impedido su formación en todos los $\operatorname{casos}^{2,28}$. Además, se ha visto que pueden infligir un daño considerable a las anastomosis en contacto y, por lo tanto, no se recomienda su uso en tales $\operatorname{casos}^{1,28}$. Se ha encontrado una menor eficiencia utilizando gel y barreras líquidas de estos compuestos $^{12}$.

Los glucocorticoides también se han estudiado, solos y en combinación con antihistamínicos; es conocido que los glucocorticoides disminuyen la respuesta inflamatoria, mientras que los antihistamínicos inhiben la proliferación de fibroblastos ${ }^{2,9}$. No se ha encontrado ningún beneficio significativo con el uso de glucocorticoides (intravenosos, entéricos o intraperitoneales) para evitar la formación de adherencias $^{9}$, y además producen efectos adversos, tales como inmunosupresión, y retrasan la reparación de la herida quirúrgica².

Se ha propuesto el uso de antibióticos para disminuir la formación de $A A P^{29,30}$, bajo el principio de que al prevenir la infección intraperitoneal puede evitarse el proceso de la inflamación y así lograr una menor formación de AAP. Según Sortini, et al. ${ }^{22}$, la instilación intraperitoneal de antibióticos promueve la formación de AAP.

El tPA es la proteína que activa a la plasmina para iniciar el proceso de fibrinólisis, y en consecuencia impide la organización y la maduración de tejido cicatricial. Se ha estudiado en un modelo experimental en conejos ${ }^{31,32}$. No obstante, la concentración requerida para evitar la formación de adherencias aumenta el riesgo de hemorragia postoperatoria y retrasa la reparación de la herida quirúrgica, y por lo tanto su uso es considerado inseguro e ineficaz ${ }^{2,9}$.

Algunos estudios han sugerido el uso de azul de metileno, un inhibidor de los radicales libres de oxígeno y por lo tanto de la respuesta inflamatoria local y sistémica, con el objetivo de disminuir la formación de adherencias ${ }^{33}$. En el estudio experimental de Dinc, et al. ${ }^{34}$ en un modelo en ratones se encontró una disminución de la expresión de óxido nitroso e hidroxiprolina, con una menor formación temprana de adherencias (7 días) en comparación con la instilación de solución salina, pero con una mayor predisposición a lesionar una anastomosis entérica.

Hay estudios en los que algunas sustancias, aparentemente no relacionadas con la génesis de las AAP, tienen efectos sobre la prevención de su formación. Así, en el laboratorio de investigación quirúrgica de la Facultad de Medicina de la Universidad de Boston ${ }^{28,35}$ se encontraron medicamentos antieméticos que contienen antagonistas de los receptores de la neurocinina-1 (aprepitant, Emend ${ }^{\circledR}$ y otros todavía en investigación), que han demostrado ser eficaces en la reducción de la formación de AAP, sobre todo cuando se aplican directamente sobre el peritoneo, pero se requieren estudios confirmatorios ${ }^{36,37}$.

\section{Conclusiones}

Las AAP son causa de dolor abdominal crónico, obstrucción intestinal e infertilidad en las mujeres. Hasta ahora, el tratamiento consiste en la liberación quirúrgica 
de las adherencias, que a su vez va acompañado de un aumento del riesgo de recurrencia. La prevención de la formación de las AAP está encaminada a disminuir el daño mesotelial con el manejo gentil de los tejidos y a evitar el uso exagerado del electrocauterio y de material de sutura; los estudios experimentales están orientados hacia la limitación de los procesos de inflamación y de la cascada de la coagulación, limitando la formación de fibrina y colágena. El uso clínico de anticoagulantes, antinflamatorios e inmunomoduladores aún no ha tenido resultados satisfactorios.

\section{Conflicto de intereses}

Los autores declaran no tener ningún conflicto de intereses.

\section{Financiamiento}

Ninguno.

\section{Responsabilidades éticas}

Protección de personas y animales. Los autores declaran que para esta investigación no se han realizado experimentos en seres humanos ni en animales.

Confidencialidad de los datos. Los autores declaran que en este artículo no aparecen datos de pacientes.

Derecho a la privacidad y consentimiento informado. Los autores declaran que en este artículo no aparecen datos de pacientes.

\section{Bibliografía}

1. Arung W, Meurisse M, Detry O. Pathophysiology and prevention of postoperative peritoneal adhesions. World J Gastroenterol. 2011; 17:4545-53.

2. Ergul E, Korukluoglu B. Peritoneal adhesions: facing the enemy. Int $J$ Surg. 2008;6:253-60.

3. Brüggmann D, Tchartchian G, Wallwiener M, Münstedt K, Tinneberg HR, Hackethal A. Intra-abdominal adhesions. Dtsch Arztebl Int. 2010; 107:769-75.

4. Liakakos T, Thomakos N, Fine P, Dervenis C, Young L. Peritoneal adhesions: etiology, pathophysiology and clinical significance. Dig Surg. 2001;18:260-3.

5. Correa-Rovelo JM, Villanueva-López GC, Medina-Santillán R, Carrillo-Esper R, Díaz-Girón-Gidi A. Obstrucción intestinal secundaria a formación de adherencias postoperatorias en cirugía abdominal. Revisión de la literatura. Cir Cir. 2015;83:345-51.

6. Maciver A, McCall M, Shaphiro J. Intra-abdominal adhesion: cellular mechanisms and strategies for prevention. Int J Surg. 2011;9:589-94.

7. Isaza-Restrepo A, Martín-Saavedra JS, Vélez-Leal JL, Vargas-Barato F, Riveros-Dueñas $R$. The peritoneum: beyond the tissue - a review. Front Physiol. 2018;9:738.

8. Butureanu SA, Butureanu TA. Pathophysiology of adhesions. Chirurgia. 2014;109:293-8.

9. Pados G, Venetis CA, Almaloglou K, Tarlatzis BC. Prevention of intra-peritoneal adhesions in gynaecological surgery: theory and evidence. Reprod Bio Med Online. 2010;21:290-303.

10. Capobianco A, Cottone L, Monno A, Manfredi AA, Rovere-Querini P. The peritoneum: healing, immunity, and diseases. J Pathol. 2017;243:137-47.
11. Sulaiman H, Dawson L, Laurent G, Belligan G, Herrick S. Role of plasminogen activators in peritoneal adhesion formation. Biochem Soc Trans. 2002;30:126-31.

12. Ward BC, Panitch A. Abdominal adhesions: current and novel therapies. J Surg Res. 2011;165:91-111.

13. Nappi C, Spiezio Sardo A, Greco E, Guida M, Bettocchi S, Bifulco G. Prevention of adhesions in gynaecological endoscopy. Hum Reprod. 2007;13:379-94.

14. Jasper V, Jan B, Roberta C, Tjalina H, Greet M, Michelle N, et al. Belgian consensus on adhesion prevention in hysteroscopy and laparoscopy. Gynecol Surg. 2015;12:179-88.

15. Vrijland WW, Jeekel, van Geldrop HJ, Swank DJ, Bonjer HJ. Abdominal adhesions: intestinal obstruction, pain, and infertility. Surg Endosc. 2003;17:1017-22.

16. Gutt CN, Oniu T, Schemmer $P$, Mehrabi A, Büchler MW. Fewer adhesions induced by laparoscopic surgery? Surg Endosc. 2004;18:898-906.

17. Kavic S, Kavic SM. Adhesions and adhesiolysis: the role of laparoscopy. JSLS. 2002;6:99-109.

18. Yong IK. Comparative study for preventive effects of intra-abdominal adhesion using cyclo-oxygenase-2 enzyme (COX-2) inhibitor, low molecular weight heparin (LMWH), and synthetic barrier. Yonsei Med J. 2013; 54:14971-7.

19. Wei G, Chen X, Wang G, Jia P, Xu Q, Ping G, et al. Inhibition of ciclooxigenase-2 prevents intra-abdominal adhesions by decreasing activity of peritoneal fibroblasts. Drug Des Devel Ther. 2015:9:3083-98.

20. Shahzmani $P$, Ashrafzadeh $M$, Jahanshahi A, Sotoudeh A. Effects of dexamethasone, piroxicam and sterile Aloe vera extracts on prevention 0 postoperative peritoneal adhesion formation in rat. Adv Environ Biol. 2012;6:2851-65

21. Guvental T, Cetin A, Ozdemir H, Yanar O, Kaya T. Prevention of postoperative adhesion formation in rat uterine horn model by nimesulide: a selective COX-2 inhibitor. Hum Reprod. 2001;16:1732-5.

22. Sortini D, Feo CV, Maravegias K, Carcoforo P, Pozza E, Liboni A, et al. Role of peritoneal lavage in adhesion formation and survival rate in rats: an experimental study. J Invest Surg. 2006;19:291-7.

23. Kement M, Censur Z, Oncel M, Buyukokuroglu M, Gezen F. Heparin for adhesion prevention: comparison of three different dosage with separafilm in a murine model. Int J Surg. 2011;9:225-8.

24. Sharifi S, Derakshanfar A, Pourjafar M, Mohamadnia A, Charlang K. Effect of heparin in prevention of experimental abdominal adhesions in rat. Iranian J Vet Surg. 2007;2:24-31.

25. Kutlay J, Ozer Y, Isik B, Kargici H. Comparative effectiveness of several agents for preventing postoperative adhesions. Worl J Surg. 2004;28:662-5.

26. Beck DE, Cohen Z, Fleshman JW, Kaufman HS, van Goor H, Wolff BG. A prospective, randomized, multicenter, controlled study of the safety of Seprafilm(R) adhesion barrier in abdominopelvic surgery of the intestine. Dis Colon Rectum. 2003;46:1310-9.

27. ten Kate-Booij MJ, van Geldorp HJ, Drogendijk AC. Dextran and adhesions in guinea-pigs. J Reprod Fert. 1985;75:183-8.

28. Cassidy RM, Sherburne AC, Heydrick SJ, Stucchi AF. Combined intraoperative administration of a histone deacetylase inhibitor and a neurokin-1 receptor antagonist synergistically reduces intra-abdominal adhesion formation in a rat model. Surgery. 2015;157:581-9.

29. Rappaport WD, Holcomb M, Valente J, Chvapil M. Antibiotic irrigation and the formation of intraabdominal adhesions. Am J Surg. 1989;158:435-7.

30. Jallouli M, Hakim A, Znazen A, Chaabouni Y, Sahnoun Z, Hammami A, et al. The role of neutrophils in the formation of peritoneal adhesions. Tunis Med. 2012;90:730-4.

31. Doody KJ, Dunn RC, Buttram VC Jr. Recombinant tissue plasminogen activator reduces adhesion formation in a rabbit uterine horn model. Fertil Steril. 1989;51:509-12.

32. Menzies $D$, Ellis $H$. The role of plasminogen activator in adhesion prevention. Surg Gynecol Obstet. 1991;172:362-6.

33. Yildiz H, Durmus AS, Simsek H, Yaman I. The comparison of methylene blue and vitamin $\mathrm{E}$ in prevention of abdominal postoperative adhesion formation in rat uterine horn models: biochemical and histopathologic evaluation. Acta Cir Bras. 2011;26:51-7.

34. Dinc S, Ozaslan C, Kuru B, Karaca S, Ustun H, Alagol H, et al. Methylene blue prevents surgery-induced peritoneal adhesions but impairs the early phase of anastomotic wound healing. Can J Surg. 2006;49:321-8.

35. Reed KL, Fruin AB, Bishop-Bartolomei KK, Gower AC, Nicolaou M, Stucchi AF, et al. Neurokin-1 receptor and substance $P$ messenger RNA levels increase during intrabdominal adhesion formation. J Surg Res. 2002;108:165-72.

36. Prushik SG, Aarons CB, Matteotti R, Reed KL, Gower AC, Leeman SE, et al. A neurokinin 1 receptor antagonist decreases adhesion reformation after laparoscopic lysis of adhesions in a rat model of adhesion formation. Surg Endosc. 2007;21:1790-5.

37. Lim R, Morrill JM, Prushik SG, Reed KL, Gower AC, Leeman SE, et al. An FDA approved neurokinin-1 receptor antagonist is effective in reducing intraabdominal adhesions when administered intraperitoneally, but not orally. J Gastrointest Surg. 2008;12:1754-61. 